\title{
Anti-adaptors provide multiple modes for regulation of the RssB adaptor protein
}

\author{
Aurelia Battesti, Joel R. Hoskins, Song Tong, Paola Milanesio, ${ }^{1}$ Jessica M. Mann, ${ }^{2}$ Andrea Kravats, ${ }^{3}$ \\ Yodit M. Tsegaye, ${ }^{4}$ Alexandre Bougdour, ${ }^{5}$ Sue Wickner, and Susan Gottesman ${ }^{6}$ \\ Laboratory of Molecular Biology, National Cancer Institute, Bethesda, Maryland 20892, USA
}

\begin{abstract}
RpoS, an RNA polymerase $\sigma$ factor, controls the response of Escherichia coli and related bacteria to multiple stress responses. During nonstress conditions, RpoS is rapidly degraded by ClpXP, mediated by the adaptor protein RssB, a member of the response regulator family. In response to stress, RpoS degradation ceases. Small anti-adaptor proteins-IraP, IraM, and IraD, each made under a different stress condition-block RpoS degradation. RssB mutants resistant to either IraP or IraM were isolated and analyzed in vivo and in vitro. Each of the anti-adaptors is unique in its interaction with RssB and sensitivity to RssB mutants. One class of mutants defined an RssB $\mathrm{N}$-terminal region close to the phosphorylation site and critical for interaction with IraP but unnecessary for IraM and IraD function. A second class, in the RssB C-terminal PP2C-like domain, led to activation of RssB function. These mutants allowed the response regulator to act in the absence of phosphorylation but did not abolish interaction with anti-adaptors. This class of mutants is broadly resistant to the anti-adaptors and bears similarity to constitutively activated mutants found in a very different PP2C protein. The mutants provide insight into how the anti-adaptors perturb RssB response regulator function and activation.
\end{abstract}

[Keywords: RpoS; ClpX; IraP; response regulator; RsbP]

Supplemental material is available for this article.

Received August 29, 2013; revised version accepted November 15, 2013.

Depending on the environmental conditions and the availability of nutrients, bacteria modulate the balance between growth and survival. In Escherichia coli, this process includes the use of specialized $\sigma$ factors that provide promoter specificity to RNA polymerase. In favorable growth conditions, RpoD is the most abundant $\sigma$ factor. RpoS is an alternative $\sigma$ factor produced in response to a variety of stress conditions and in stationary phase (for review, see Battesti et al. 2011). This protein is highly regulated at all levels, including the level of protein stability. RpoS is degraded by the ATPdependent protease ClpXP. However, for it to be recognized as a substrate, RpoS must first interact with the adaptor protein RssB. RssB is an orphan atypical response regulator. Its $\mathrm{N}$-terminal domain is characteristic of the large family of response regulator proteins and, like other response regulators, can be phosphorylated on a conserved

Present addresses: ${ }^{1} 73$ Farrer Drive \#09-02, Sommerville Park, Singapore 259280, Singapore; ${ }^{2}$ National Institute of Allergy and Infectious Disease, Building 4, Room 109, Bethesda, MD 20892, USA; ${ }^{3}$ Department of Chemistry, Box 210172, University of Cincinnati, Cincinnati, OH 45221 , USA; ${ }^{4}$ George Washington School of Medicine and Health Sciences, 2300 I Street NW, Washington, DC 20037, USA; ${ }^{5}$ UMR 5163, Laboratoire Adaptation et Pathogénie des Microorganismes, Centre National de la Recherche Scientifique, 38041 Grenoble, France.

${ }^{6}$ Corresponding author

E-mail gottesms@helix.nih.gov

Article is online at http://www.genesdev.org/cgi/doi/10.1101/gad.229617.113. aspartic acid residue (D58 in RssB). It is unclear what kinases or small molecules are necessary for RssB phosphorylation; in vitro, acetyl phosphate (AcP) can phosphorylate RssB and activate it. Mutants unable to be phosphorylated retain a significant amount of activity (Peterson et al. 2004; for review, see Hengge 2009). In contrast to most other response regulators, the C-terminal domain of RssB does not contain a DNA-binding domain. Instead, the C terminus has similarities to PP2C Ser/Thr phosphatases but lacks a phosphatase active site (Galperin 2006, 2010).

RpoS degradation occurs during favorable growth conditions, when RpoS is not needed. The inhibition of RpoS degradation during stress conditions allows RpoS levels to rise, activating RpoS-dependent genes. In response to specific stress treatments, RpoS is stabilized by the inhibition of RssB activity by small proteins called antiadaptors. Three anti-adaptor proteins have been described and named IraP, IraM, and IraD (Bougdour et al. 2006, 2008). These three proteins are induced under different stress conditions: phosphate starvation for IraP (Bougdour

This article is distributed exclusively by Cold Spring Harbor Laboratory Press for the first six months after the full-issue publication date (see http://genesdev.cshlp.org/site/misc/terms.xhtml). After six months, it is available under a Creative Commons License (Attribution-NonCommercial 3.0 Unported), as described at http://creativecommons.org/licenses/ by-nc/3.0/. 
et al. 2006), magnesium starvation for IraM (Bougdour et al. 2008), and DNA damage or transition from exponential to stationary phase for IraD (Bougdour et al. 2008; Merrikh et al. 2009a,b). Despite a common function and common small size, IraP, IraM, and IraD are not members of the same protein family and do not share sequence similarity.

Here we used a genetic approach to study the interaction of the anti-adaptor proteins with RssB, isolating mutations in RssB resistant to anti-adaptor action. We found that each of the anti-adaptors is unique in its interaction with RssB and its sensitivity to mutants in RssB. Mutations in the C-terminal PP2C domain activate RssB and are similar to those observed to activate a very different bacterial PP2C protein. The interactions of antiadaptors and RssB highlight ways in which other members of the large family of response regulators may be modulated by small proteins.

\section{Results}

Anti-adaptor proteins interact with different regions of RssB

Previous in vitro work demonstrated that anti-adaptor proteins inhibit degradation of RpoS by ClpXP and physically interact with RssB (Bougdour et al. 2006, 2008). This suggested that the anti-adaptors may inhibit degradation by occluding sites on RssB required for the normal adaptor function of RssB. To test this possibility, we wanted to identify the regions of RssB that interact with each of the three anti-adaptor proteins, IraP, IraD, and IraM. We used a bacterial two-hybrid assay in which the two domains of the Bordetella pertussis Cya (cyclase) protein, T18 and T25, are fused to the proteins to be tested. A positive interaction leads to synthesis of cyclic AMP, assayable in an E. coli cya host by measuring expression of $\beta$-galactosidase (Karimova et al. 1998; Battesti and Bouveret 2012).

We cloned the intact or truncated portions of the rss $B$ coding region as well as each of the anti-adaptor genes in frame with the $\mathrm{N}$-terminal T18 or T25 fragments of cya in compatible plasmids (Gully and Bouveret 2006). Endpoints for the truncated RssB derivatives were chosen based on the known boundaries of the response regulator domain (Volz 1995) and a structure for an RssB-related protein from Pseudomonas aeruginosa (Protein Data Bank [PDB]: 3EQ2) (Fig. 1A). The resulting recombinant IraP, IraM, IraD, and full-length RssB fusion proteins were functional (Supplemental Fig. S1). Fusion proteins with deletions of either the $\mathrm{N}$-terminal or C-terminal domain of RssB were expressed at similar levels (Supplemental Fig. S1C) and, as expected, were inactive for degradation of RpoS (Klauck et al. 2001; our unpublished results).

In control experiments, we detected an interaction between wild-type RssB and each of the three antiadaptor proteins; there was no interaction of the antiadaptors with the T18 fragment alone (vector control) (Fig. 1B, RssB WT bars [full-length RssB] set to $100 \%$ compared with vector [no RssB]). IraP and IraD both showed interactions with the truncated proteins containing the RssB $\mathrm{N}$-terminal response regulator domain but not with those containing the C-terminal domain (Fig. $1 \mathrm{~B}$, left and middle panels). IraM, however, interacted with the fusion proteins containing the RssB C-terminal domain $\left(\mathrm{C}_{124-337}\right.$ and $\left.\mathrm{C}_{160-337}\right)$ (Fig. 1B) as well as the fusion containing the $\mathrm{N}$-terminal domain and the linker $\left(\mathrm{N}_{1-160}\right)$ but not the fusion containing the $\mathrm{N}$-terminal domain alone $\left(\mathrm{N}_{1-124}\right)$ (Fig. 1B, right panel).

When the RssB fusion proteins were expressed from the lower copy number T25 vector, the pattern of interactions was the same. However, RssB N-terminal domain interactions with IraM were significantly improved if the host strain was deleted for rpoS (Supplemental Table S1). This suggests that the bacterial two-hybrid system is sensitive to expression of host proteins-in this case, RpoS-and that interactions of the anti-adaptors with RssB domains are not as strong as for the full-length RssB and thus can be competed by RpoS. Note that in the strains expressing functional anti-adaptor fusion proteins, RpoS is stabilized and therefore accumulates (Supplemental Fig. S1B).

We also monitored interactions between the antiadaptor proteins and either RssB full-length protein or truncated RssB derivatives (Fig. 1A) in pull-down assays with calmodulin-coated beads. The CBP /calmodulinbinding peptide) tag was fused to the $\mathrm{N}$ terminus of wild-type RssB and to each of the RssB fragments (Fig. 1A). The resulting full-length CBP-RssB fusion protein was functional (Supplemental Fig. S1A). Lysates of cells expressing each of the CBP fusions and one of the three anti-adaptors were used in the pull-down assays; the presence of anti-adaptors associated with the CBP fusion proteins was determined by Western blot analysis.

The three anti-adaptors associated with full-length RssB (Fig. 1C, RssB WT lanes), while no interaction was observed between the anti-adaptors and CBP alone (Fig. $1 \mathrm{C}$, vector lanes). Consistent with the results obtained in the two-hybrid experiments, IraP and IraD associated with RssB $\mathrm{N}_{1-124}$ and $\mathrm{N}_{1-160}$ fusions containing the response regulator domain of RssB but not with fusions to the C-terminal domain. IraM associated with the C-terminal domain (Fig. 1C), suggesting that the interaction with the $\mathrm{C}$-terminal domain is stronger than that with the $\mathrm{N}$-terminal domain $\left(\mathrm{N}_{1-160}\right)$ detected by the bacterial two-hybrid assay. These results are most consistent with the data from the rpoS $S^{+}$bacterial two-hybrid assay. The experiments demonstrate that the anti-adaptor proteins interact with RssB in at least two different ways, with IraP and $\mathrm{IraD}$ both interacting with the well-conserved response regulator domain, and IraM interacting with the $\mathrm{C}$-terminal domain and, more weakly, the $\mathrm{N}$-terminal domain.

We suggest that anti-adaptors interfere with RssBRpoS interaction directly or indirectly. In the absence of ClpXP activity, RssB acts as an anti- $\sigma$, inhibiting RpoSdependent transcription (Zhou and Gottesman 1998; Becker et al. 2000). Blocking the interaction of RpoS with RssB would free RpoS to carry out transcription, thus allowing the RpoS-dependent response to the stress that 
Battesti et al.
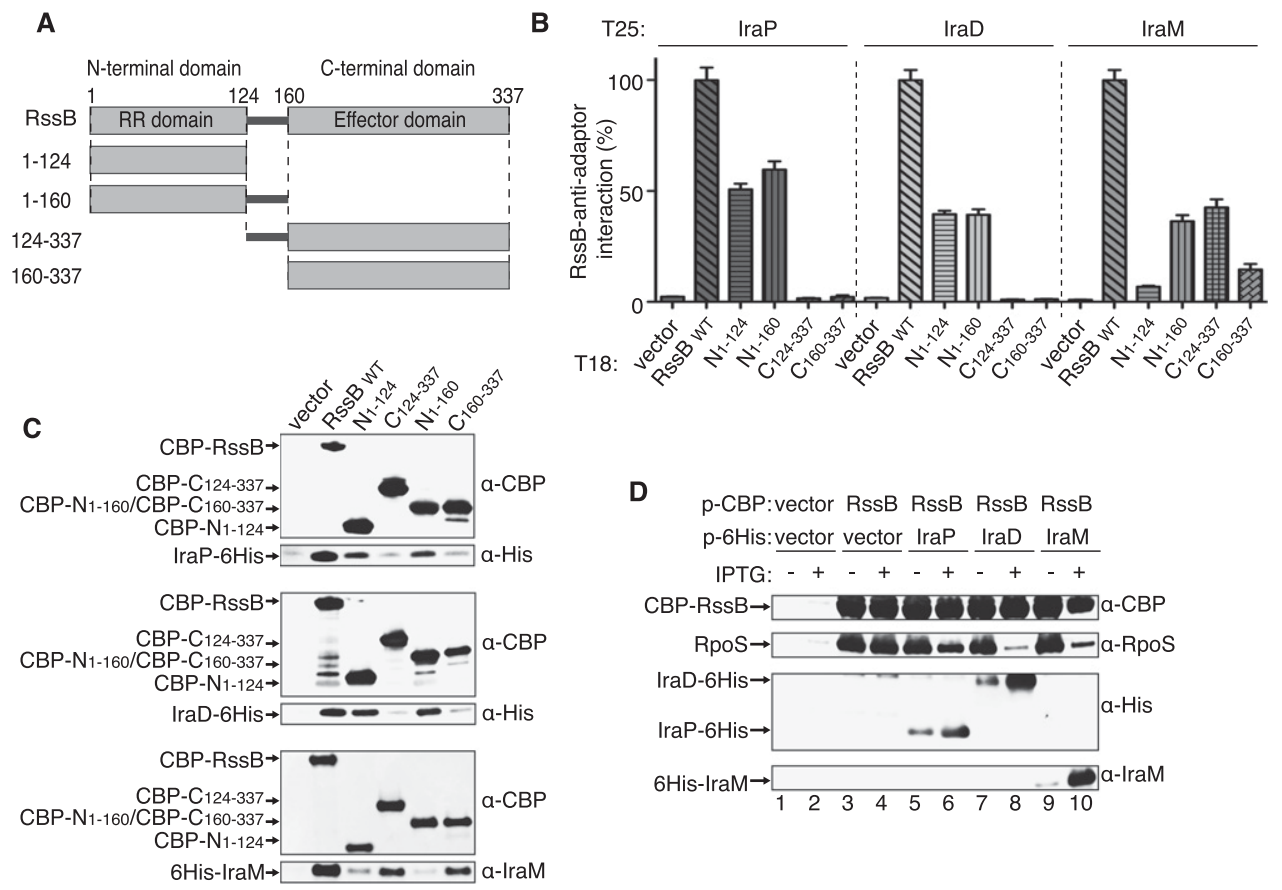

Figure 1. Interaction between RssB and anti-adaptor proteins. $(A)$ Schematic representation of the truncated RssB proteins. $(B)$ Interaction of RssB domains with anti-adaptors by bacterial two-hybrid assay. The indicated RssB domains fused to the T18 subunit were assayed for interaction with the anti-adaptor proteins IraP, IraD, and IraM fused to the T25 domain in BTH101. Interaction was assayed by $\beta$-galactosidase assay (see the Materials and Methods). The interaction between RssB wild-type (WT) and anti-adaptor proteins was set at $100 \%$ for each series. Mean values obtained for each interaction with the full-length (FL) RssB (Miller units): T25IraP/T18-RssB, 1637; T25-IraD/T18-RssB, 1564; and T25-IraM/T18-RssB, 2835. The pattern of interaction was similar for assays done with the T18-anti-adaptors and T25-RssB constructs (Supplemental Table S1). (C) Interaction of the truncated RssB proteins with the anti-adaptors by pull-down assay. Pull-down experiments on calmodulin beads were performed on extracts from MG1655 cotransformed with plasmids containing CBP-tagged RssB domains and pQE plasmids containing the anti-adaptor proteins with His tags (Bougdour et al. 2006, 2008). CBP and pQE plasmids are listed in Supplemental Table S7. Cells were grown, and samples were taken and processed as described in the Materials and Methods and Supplemental Material. For IraP, sample loading volume was four times higher for the domain samples than for the RssB full-length (wild type [WT]) samples. $(D)$ Competition between RpoS and the antiadaptors for RssB binding. Experiments were similar to $C$, with the same sets of plasmids as for $C$ transformed into a MG1655 clpX:: kan strain (BA183). Cells were grown at $37^{\circ} \mathrm{C}$ in $\mathrm{LB}$ (Lennox broth) to $\mathrm{OD}_{600}=0.7$, and $0.05 \%$ arabinose was added for $1 \mathrm{~h}$ to induce production of the CBP-tagged proteins. Each culture was then divided in two; one-half was pelleted, and the other half was induced with $0.5 \mathrm{mM}$ IPTG for $20 \mathrm{~min}$ to induce the production of the anti-adaptor proteins. Pull-down samples were analyzed by Western blot using anti-CBP, anti-RpoS, anti-His, and anti-IraM antisera.

leads to anti-adaptor induction. We tested this model in vivo by overproducing specific anti-adaptors and assaying their effect on the interaction of RssB with RpoS (Fig. 1D). MG1655 clpX::kan was cotransformed with the plasmid expressing the CBP-RssB full-length protein and a plasmid producing one of the anti-adaptor proteins under IPTG control. As expected, the $\operatorname{clpX}$ deletion stabilized RpoS so that RpoS levels were the same in all extracts (Supplemental Fig. S1D). CBP-RssB was isolated from cell lysates by calmodulin affinity purification both before and after induction of the anti-adaptors and assayed for associated RpoS and anti-adaptors by Western blot (Fig. 1D). In the absence of anti-adaptors, similar amounts of RpoS associated with full-length RssB before and after IPTG addition (Fig. 1D, cf. lanes 3 and 4). IPTG induction of each of the anti-adaptors was sufficient to decrease the amount of RpoS associated with CBP-RssB (Fig. 1D, IraP [cf. lanes 5 and 6, IraD [cf. lanes 7 and 8], and IraM [cf. lanes 9 and 10]). These results support our model that the anti-adaptor proteins interfere with the ability of RpoS to bind RssB.

\section{Genetic screen for RssB mutants insensitive to IraP and IraM}

In order to better understand how the anti-adaptor proteins interact with RssB, we performed genetic screens to look for RssB mutants resistant to IraP or IraM antiadaptors. We used a strain containing a translational lacZ fusion to rpoS, expressed from an arabinose-inducible promoter. We previously showed that degradation of this RpoS-LacZ fusion protein is RssB- and ClpXP-dependent; this assay was originally used to identify the antiadaptors (Bougdour et al. 2006, 2008). Anti-adaptor expression was increased by replacing the native chromosomal iraP or iraM promoter with more active promoters (see the details in the legends for Supplemental Tables S2, S3). 
Strains expressing high levels of IraP, referred to as iraP $_{U P}$, were transformed with one of two libraries of plasmids. In one plasmid library, the $5^{\prime}$ end of $r s s B$ (encoding amino acids 1-169) was randomly mutagenized; in the second library, the $3^{\prime}$ end (encoding amino acids 170-337) was randomly mutagenized. Transformants were grown on lactose MacConkey agar plates containing ampicillin and arabinose to express the pBAD$r p o S$-lacZ fusion. On these plates, colonies containing the vector were red (RpoS-LacZ not degraded), while those expressing wild-type $\mathrm{RssB}^{+}$were dark pink (limited degradation of RpoS-LacZ) (see RssB [WT] sectors on plates in Fig. 2A; Supplemental Fig. S2A). Colonies that are lighter pink than the wild-type $\mathrm{RssB}^{+}$control would be expected to contain plasmids expressing RssB mutant proteins able to degrade RpoS even in the presence of high levels of IraP.
Several plasmids encoding RssB derivatives resistant to IraP were identified, sequenced, and confirmed by reintroducing the plasmid into JMM03, an $r s s B:$ :tet strain carrying the iraP $P_{\mathrm{UP} 1}$ allele and the $r p o S-l a c Z$ fusion. Many, but not all, plasmids contained more than one mutation (Supplemental Table S2). Most mutations were not obtained multiple times; therefore, the screen for mutants may not be saturated. Mutants resistant to IraP were found, as expected, in the $\mathrm{N}$-terminal domain but also in the C-terminal domain of RssB, which did not appear to bind IraP (Fig. 1). These mutants may bypass IraP inhibition or affect binding indirectly.

The multiple and single mutations were reconstructed in the $r s s B$ plasmid and compared with the original mutant plasmids (Supplemental Fig. S2B,C). Almost all plasmids expressed RssB that was functional for degrada-
A

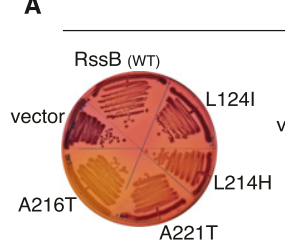

iraP $u p$ strain
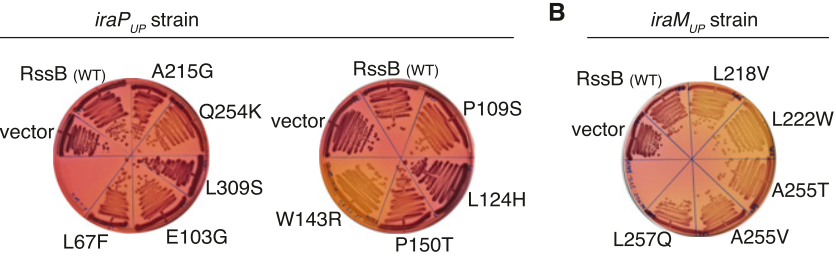

C

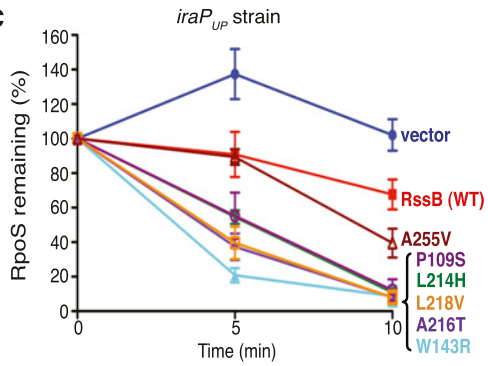

D

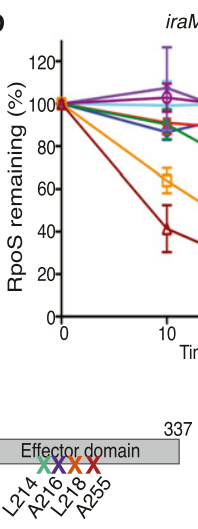

$\mathbf{F}$

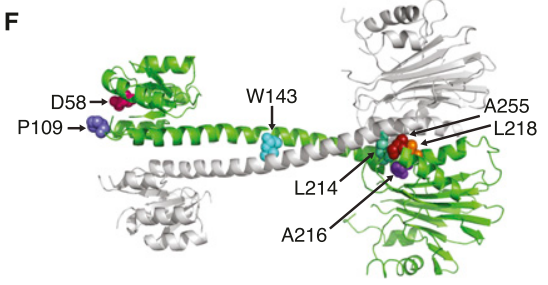

G

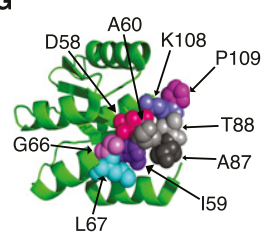

Figure 2. IraP- and IraM-resistant RssB mutants obtained by genetic screen. (A) IraP resistance of selected RssB mutants on lactose MacConkey plates. pHDB3 (vector control), pHDB3-RssB wild type (WT), and the reconstructed pHDB3-RssB mutants were transformed into JMM03 (pBAD-rpos-lacZ rssB:: tet iraP $\left.P_{\mathrm{UP} 1}\right)$, plated on lactose MacConkey plates containing $0.00002 \%$ arabinose and $100 \mu \mathrm{g} / \mathrm{mL}$ ampicillin, and incubated for $20 \mathrm{~h}$ at $30^{\circ} \mathrm{C}$. The phenotypes of the original mutants are shown in Supplemental Figure S2A. (B) IraM resistance of selected RssB mutants on lactose MacConkey plates. pHDB3 (vector control), pHDB3-RssB wild type, and pHDB3-RssB reconstructed mutants were transformed into BA290 [pBADrpos-lacZ rssB::tet zeo-cp18-iraM (iraM $\left.M_{U P 1}\right)$ ], plated on lactose MacConkey plates containing $50 \mu \mathrm{g} / \mathrm{mL}$ ampicillin, and incubated at $37^{\circ} \mathrm{C}$. The phenotypes of the original mutants are shown in Supplemental Figure S3A. (C) RpoS stability assay in an $\operatorname{iraP}_{\mathrm{UP}}$ strain. Reconstructed plasmids producing wildtype or mutated RssB were transformed into ST1011 [imm21-rpoS-lac zeo-cp18-IraP $\left(\right.$ iraP $\left._{\mathrm{UP} 2}\right)$ rssB ::tet $\Delta$ lac]. Cells were grown at $37^{\circ} \mathrm{C}$, chloramphenicol was added, and samples were removed and analyzed for RpoS as described in the Materials and Methods. The level of RpoS measured at time $=0$ for each mutant was set to $100 \%$. (D) RpoS stability assay in an iraM $_{\mathrm{UP}}$ strain. Recon-

structed plasmids producing wild-type or mutated RssB were transformed into BA290. Cells were grown, and samples were treated as for C. $(E)$ Schematic representation of RssB. The RssB N-terminal domain (amino acids 1-124) and C-terminal domain (amino acids 160-337) are connected by a linker region. Mutations selected during the screen for IraP- and IraM-resistant RssB mutants are indicated by an X. The number below each X corresponds to the mutated amino acid based on the E. coli sequence; color-coding of the $\mathrm{X}$ corresponds to that used in the graphs in $C$ and $D$, as well as in the RssB model $(F)$. $(F)$ Localization of mutations on the structure of RssB from $P$. aeruginosa. The model of the $\operatorname{RssB}_{P \text {. aeruginosa }}$ dimer was created from the RssB structure of $P$. aeruginosa RssB (PDB: 3EQ2) (I Levchenko, RA Grant, RT Sauer, and TA Baker, unpubl.) using PYMOL (http://www.pymol.org). One protomer is colored in green; residues mutated during the screen are represented as Corey-Pauling-Koltun (CPK) models. Sequence alignment of $\mathrm{RssB}_{E}$. coli and $\mathrm{RssB}_{P \text {. aeruginosa }}$ was performed using the ClustalW2 program from the EMBL-EBI Web site (http://www.ebi.ac.uk/ Tools/msa/clustalw2) with default settings. $(G)$ Crystal structure of the RssB N-terminal domain from E. coli. The RssB . coli N-terminal domain (PDB: 3EOD) was visualized using PYMOL (http://www.pymol.org). Residues on the surface close to the phosphorylation site that were mutated and tested for interaction with IraP are color-coded. Mutations in residues 60, 87, and 88 had no effect on the interaction with IraP; these are colored gray (data not shown). 
tion of RpoS in strains containing the normal levels of the anti-adaptors. Mutants were also tested in strains expressing increased levels of IraP (Fig. 2A), IraD (Supplemental Fig. S2D), or IraM (Supplemental Fig. S2E); the results of these plate tests on all original and reconstructed mutants are summarized in Supplemental Table S4. The majority of reconstructed mutants had properties similar to the original mutants; single mutants with antiadaptor-resistant properties were characterized further.

A similar screen was carried out for resistance to high levels of IraM using the library of plasmids randomly mutagenized within the $3^{\prime}$ end (encoding amino acids 170-337 of RssB). We isolated five different mutants resistant to IraM (Supplemental Fig. S3A; Supplemental Table S3). These mutants and mutants reconstructed in the $r s s B$ plasmid were tested for activity (Supplemental Fig. S3B,C) and anti-adaptor resistance (Fig. 2B, IraM; Supplemental Fig. S3D, IraP; Supplemental Fig. S3E, IraD); the results are summarized in Supplemental Table S4. Interestingly, some IraM-resistant mutations were found close to the sites mutated in the IraP-resistant isolates (Supplemental Tables S2, S4).

\section{Several mutant RssB proteins show resistance to multiple anti-adaptors}

Four mutants isolated as IraP-resistant (P109S, W143R, $\mathrm{L} 214 \mathrm{H}$, and A216T) and two mutants isolated as IraMresistant (L218V and $\mathrm{A} 255 \mathrm{~V})$ were chosen as the focus of the work reported here (Fig. 2E). Although RssB from E. coli has not been crystallized, a $P$. aeruginosa protein with sequence ( $24 \%$ identity) but not functional homology with $E$. coli RssB has been crystallized by Baker and coworkers (I Levchenko, RA Grant, RT Sauer, and TA Baker, unpubl.) (PDB: 3EQ2). In addition, they reported the structure of the N-terminal response regulator domain of E. coli RssB (PDB: 3EOD) (Fig. 2G). We used the $P$. aeruginosa structure to show the location of the six mutants used in this study (Fig. 2F). One, P109S, is in the response regulator domain; another, W143R, lies in the linker. The other four are located in the C-terminal domain and are predicted to be close to each other in the structure. Also shown in Figure 2, F and G, is D58, the conserved aspartate that is the site of phosphorylation; an RssB D58P mutant was included in some of the experiments discussed below.

The anti-adaptor resistance phenotype of these mutants was first confirmed by direct measurement of RpoS degradation in vivo in strains containing synthetic promoters expressing increased IraP (Fig. 2C) or increased IraM (Fig. 2D). In the $\operatorname{iraP}_{U P}$ strain, a plasmid expressing wild-type RssB degraded RpoS slowly (half-life > $10 \mathrm{~min}$ ). The four mutants isolated as resistant to IraP as well as one isolated as resistant to IraM (L218V) degraded RpoS more rapidly than the control strain expressing wild-type RssB (Fig. 2C). These results are consistent with the results seen on plates, thus validating the plate assays.

Two RssB mutants, A255V and L218V, isolated as IraMresistant, showed the most rapid degradation of RpoS in the strain overexpressing IraM (iraM $\left.{ }_{U P}\right)$ (Fig. 2D). The other mutants, isolated as IraP-resistant, remained IraM sensitive in both this assay and other assays discussed below, again consistent with the plate assays. We note that in this assay, RssB P109S and RssB W143R, isolated as IraP-resistant, are even more sensitive to stabilization by IraM than is the wild-type protein (Fig. 2D).

\section{In vivo anti-adaptor phenotypes of RssB mutants are recapitulated in vitro}

The six RssB mutants were purified and tested for their ability to degrade RpoS in vitro in conjunction with ClpXP with and without anti-adaptors. As seen in vivo (Supplemental Figs. S2C, S3C), all of the RssB mutants were active for RpoS degradation in vitro in the presence of AcP, with some having slightly less activity or somewhat more activity than the wild-type (Fig. 3A; Supplemental Table S5). We monitored inhibition of degradation by the anti-adaptors using a concentration that inhibited the activity of wild-type RssB by at least 90\% (Fig. 3C-E). In this assay, significantly less IraD was needed to inhibit wild-type RssB than IraP and IraM (0.18 $\mu \mathrm{M}$ vs. $1 \mu \mathrm{M})$. The basis for the reduced requirement for IraD in vitro was not further investigated here.

P109S, the only RssB mutation in the response regulator domain (Fig. 2E), was selected as resistant to IraP. Consistent with the in vivo results (Fig. 2C,D; Supplemental Fig. S2D,E), in vitro, it was partially resistant to IraP (Fig. 3C) and sensitive to IraM and IraD (Fig. 3D,E). W143R, in the linker, was resistant to both IraP and IraD (Fig. 3C,E) but sensitive to IraM (Fig. 3D), again consistent with in vivo results (Fig. 2C,D; Supplemental Fig. S2D,E). This distinction between P109S and W143R in sensitivity to IraD suggests that while both IraP and IraD interact with the N-terminal domain of RssB, they must act somewhat differently from each other. As seen in the in vivo tests (Fig. 2D), both P109S and W143R were more sensitive to IraM than was wild-type RssB.

The other four mutant RssB proteins-L214H, A216T, L218V and A255V-showed at least partial resistance to all of the anti-adaptors in vitro (Fig. 3C-E), although in vivo qualitative results differed in a few instances (for a summary, see Supplemental Table S5). Thus, C-terminal domain mutations, whether selected for IraP resistance (L214H or A216T) or IraM resistance (L218V and A255V), change RssB sensitivity to all three anti-adaptors. This shared resistance or partial resistance to anti-adaptors that interact with different domains of RssB (Fig. 1) suggests that these mutants likely act indirectly to mediate anti-adaptor resistance.

\section{Mutations in the C-terminal domain of RssB cause changes that mimic those induced by phosphorylation in the $N$-terminal domain}

RssB, like other response regulators, is phosphorylated on a conserved aspartate (D58), and its activity is greatly stimulated in vitro by $\mathrm{AcP}$, a small molecule phosphate donor (Bouché et al. 1998; Zhou et al. 2001). As mentioned above, all of the mutants selected as anti-adaptorresistant were active in the presence of AcP (Fig. 3A; 
A
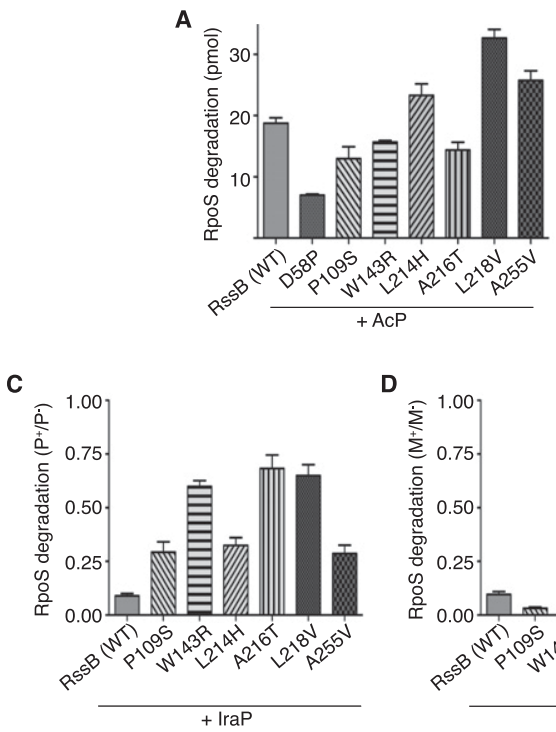

D

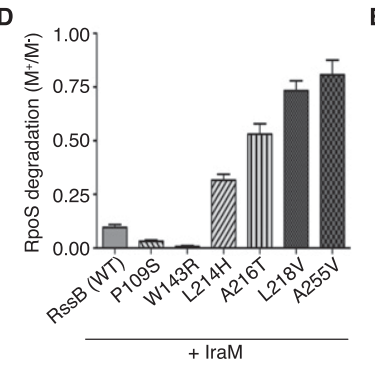

в

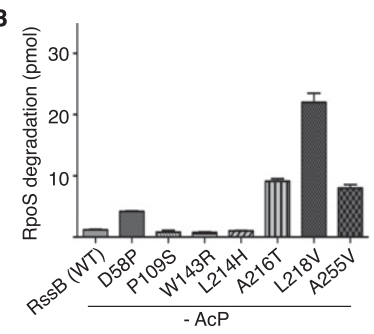

E

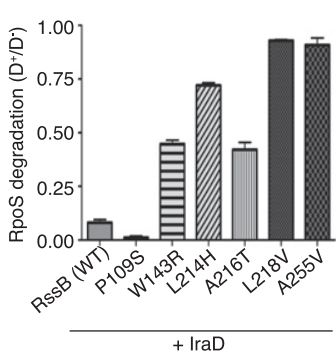

Figure 3. In vitro behavior of the RssB mutants: degradation and sensitivity to anti-adaptors. $(A, B)$ Degradation of $\left[{ }^{3} \mathrm{H}\right] \mathrm{RpoS}$ by ClpXP with RssB wild type or mutant was measured as described in the Materials and Methods in the presence $(A)$ or absence (B) of $25 \mathrm{mM}$ AcP. Data are means \pm SEM $(n \geq 3)$. D58P was used at $0.8 \mu \mathrm{M}$ (twofold above the other RssB proteins) to increase RpoS degradation to more reproducible levels. $(C-E)$ Sensitivity of the mutant RssB to anti-adaptors was measured as in $A$ in the presence of $25 \mathrm{mM}$ AcP. For each RssB protein, degradation in the absence of antiadaptors was set to 1 . Thus, a value of 1 indicates full resistance to the tested antiadaptor; 0.05 indicates $95 \%$ inhibition and therefore sensitivity to the anti-adaptor. Note that the level of RpoS degradation without anti-adaptors differs between mutants (shown in $A$; Supplemental Table S5). The levels of the anti-adaptors used in vitro were chosen to give at least $90 \%$ inhibition of the wild-type protein: $1 \mu \mathrm{M}$ for $\operatorname{IraP}(C), 1 \mu \mathrm{M}$ for $\operatorname{IraM}(D)$, and $0.18 \mu \mathrm{M}$ for $\operatorname{IraD}(E)$. Data are means \pm $\operatorname{SEM}(n \geq 3)$.

summarized in Supplemental Table S5). Surprisingly, three of the mutants-A216T, L218V, and A255V-were partially active in the absence of AcP, although AcP stimulated their activity further (Fig. 3A,B). As expected, the unphosphorylatable D58P mutant exhibited low activity with or without AcP (Fig. 3A,B, note that the level of RssB D58P used was twice that of the other proteins). These results suggest that these mutations in the C-terminal domain at least partially mimic the effect of AcP in activation of RssB.

The ability of the RssB mutants to be phosphorylated by AcP was directly tested. Purified wild-type and mutant RssB proteins were incubated with $\mathrm{AcP}$ and assayed by phosphate affinity SDS-PAGE (Phos-tag) (Fig. 4A). In the absence of AcP, one band was detected for wild-type RssB, whereas in the presence of AcP, two distinct bands were visible, indicative of the phosphorylated and unphosphorylated forms of wild-type RssB (Fig. 4A). For the $\mathrm{N}$-terminal D58P mutant, only a single band was seen in the presence or absence of $\mathrm{AcP}$, as expected; the RssB mutant at L67A, close to the site of phosphorylation (Fig. 2G), also did not show evidence of phosphorylation (Fig. 4A). However, P109S was phosphorylated, although at a lower level than seen for wild-type RssB (Fig. 4A). This is consistent with stimulation of P109S activity by AcP (Fig. 3A, 3B). All of the other mutants were phosphorylated at least as well as the wild-type protein (Fig. 4A). Therefore, mutations like $\mathrm{L} 218 \mathrm{~V}$, while relatively independent of AcP for activity (Fig. 3B), are still phosphorylated by AcP (Fig. 4A).

Altogether, the results support the idea that regulation of RpoS degradation by anti-adaptors is not via regulation of phosphorylation or dephosphorylation of RssB and is likely independent of phosphorylation. This is confirmed by two further sets of experiments. While RpoS degradation was significantly less in the absence of AcP, it was still sensitive to the anti-adaptors (Supplemental Fig. S4). In addition, the RssB mutant in the site of phosphorylation (D58P) was still sensitive to all three anti-adaptors in vitro (Supplemental Fig. S5A), consistent with in vivo experiments (Supplemental Fig. S5B; Bougdour et al. 2006, 2008).

We next tested for the ability of the RssB mutants to form stable complexes with RpoS using an indirect assay. In a previous study, we observed that RssB was protected from inactivation by the alkylating agent $\mathrm{N}$-ethylmaleimide (NEM) in the presence of RpoS and AcP (Zhou et al. 2001). Purified RssB proteins were incubated with RpoS and AcP for $5 \mathrm{~min}$; this mixture was then treated with NEM. After 10 min, NEM was inactivated, and RssB activity was determined by measuring RpoS degradation by ClpXP (Fig. 4B).

Wild-type RssB was well protected (55\% of full activity) (Fig. 4B, cf. lanes 4 and 5); protection depended completely on both AcP and RpoS (Fig. 4B, cf. lanes 2,3 and lane 4), as previously seen (Zhou et al. 2001). RssB D58P and L67A were sensitive to NEM, consistent with AcP not being able to phosphorylate these proteins. Together, these results suggest that phosphorylation is necessary for protection. While there are multiple cysteines in RssB, one likely target for NEM inactivation is Cys57, a conserved residue adjacent to the site of phosphorylation.

The results for the full set of RssB mutants are in Figure 4B. The N-terminal domain mutant P109S was the only mutant that was NEM-sensitive but able to be phosphorylated (Fig. 4A,B). Because it can be phosphorylated by AcP, although to a much lower extent than the wild-type protein (Fig. 4A) and requires AcP for RpoS degradation (Fig. 3A,B), the NEM sensitivity of P109S suggests that 
A

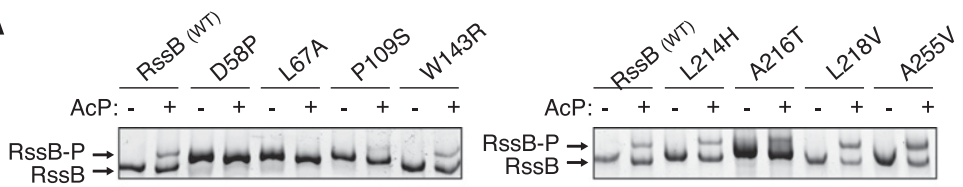

B
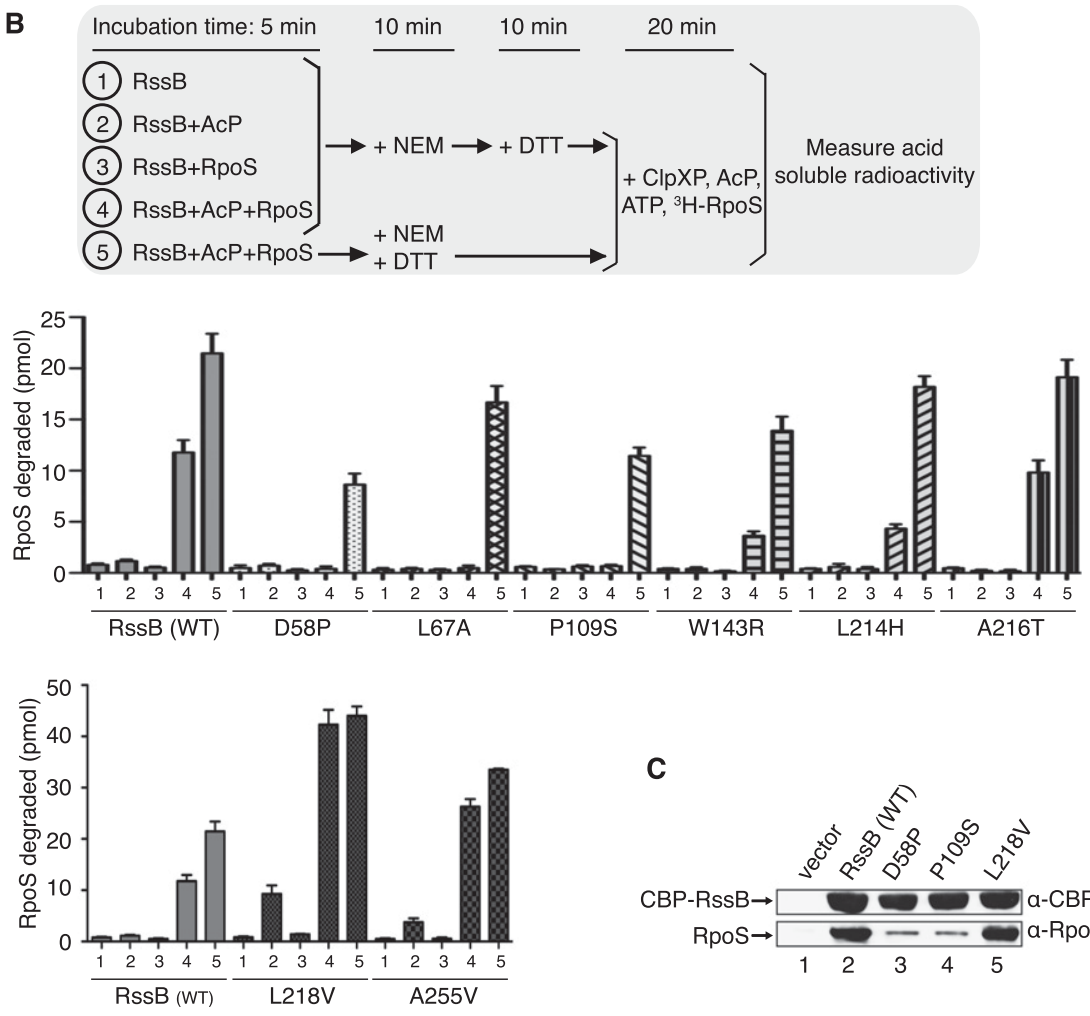

the phosphorylated RpoS-bound state of P109S is less stable than that of the wild-type protein. Alternatively, phosphorylation, while necessary for NEM resistance, may not be sufficient.

Mutants in the linker (W143R) or C-terminal domain were NEM-resistant in the presence of RpoS and AcP, and none were NEM-resistant in the absence of AcP (Fig. 4B, lane 3 in each group), consistent with a requirement for phosphorylation for NEM resistance. However, two of the C-terminal domain mutants, L218V and A255V, had modest but reproducible levels of NEM resistance after treatment with AcP in the absence of RpoS $121 \%$ of full protection for $\mathrm{L} 218 \mathrm{~V}$ and $11.2 \%$ of full protection for A255V vs. $5.6 \%$ for wild-type protein) (Fig. 4B). In addition, while wild-type RssB was $55 \%$ protected from NEM inactivation by RpoS and AcP, L218V and A255V were almost fully protected from NEM $196 \%$ for L218V; $78 \%$ for $\mathrm{A} 255 \mathrm{~V})$. Thus, these mutants are in the activated form based on two different criteria: ability to degrade RpoS in the absence of AcP (Fig. 3B) and resistance to NEM in the absence of RpoS (Fig. 4B).

Several of the RssB mutants were also tested for interaction with RpoS by a pull-down assay, carried out in a $c l p X$ mutant strain so that RpoS is stable (Fig. 4C). In
Figure 4. Phosphorylation, NEM resistance, and RpoS interaction of RssB mutants. (A) Phosphorylation of RssB wild type (WT) and mutants in vitro: Phos-tag assay. RssB wild-type or mutant proteins $(2.5 \mu \mathrm{M})$ were incubated in the presence or absence of $20 \mathrm{mM} \mathrm{AcP}$ for $30 \mathrm{~min}$ at room temperature. Samples were then analyzed by Phos-tag SDS-PAGE and stained with Coomassie, as described in the Materials and Methods. (B) NEM resistance of RssB wildtype and mutants. The ability of AcP and RpoS to protect RssB from NEM inactivation was determined as described in the Materials and Methods. Wild-type or mutant RssB was incubated in the presence or absence of AcP and/or RpoS prior to reaction with NEM. NEM was subsequently quenched with DTT, and RssB activity was assessed by measuring $\left[{ }^{3} \mathrm{H}\right] \mathrm{RpoS}$ degradation by ClpXP. The positive control was RpoS degradation activity when NEM and DTT were added together (shown in lane 5 for each set). Data are means \pm SEM $(n \geq 3)$. (C) Interaction between RssB mutants and RpoS. CBP-RssB wild-type and mutant proteins were expressed in BA183 (clpX::kan) and purified on calmodulin beads as described for Figure 1D. Pull-down samples were analyzed by Western blot using anti-CBP and anti-RpoS antisera. this assay, wild-type RssB interacted well with RpoS (Fig. 4C, lane 2). As suggested by previous work (Klauck et al. 2001), D58P significantly decreased the interaction with RpoS (Fig. 4C, lane 3). Consistent with the NEM results, P109S also showed decreased RpoS binding (Fig. 4C, lane 4). L218V bound RpoS similarly to wild-type RssB (Fig. $4 \mathrm{C}$, cf. lanes 2 and 5).

IraP-resistant mutants in the N-terminal domain have lost the ability to interact with IraP

The in vitro analysis of the RssB mutants confirmed that resistance to the anti-adaptors can be replicated in a simple in vitro system but did not directly address why the mutants are resistant to one or more of the antiadaptors. One possibility was that the mutants in RssB block anti-adaptor binding. As shown above, wild-type RssB interacts with each of the anti-adaptors in a bacterial two-hybrid assay (Fig. 1B) and in a pull-down assay (Fig. 1C). The RssB mutants were tested in these assays to see whether they retained their interaction with the antiadaptors (Fig. 5).

RssB P109S is IraP-resistant but is sensitive to IraD and IraM for RpoS degradation in vivo and in vitro (Figs. 2, 3; 
A

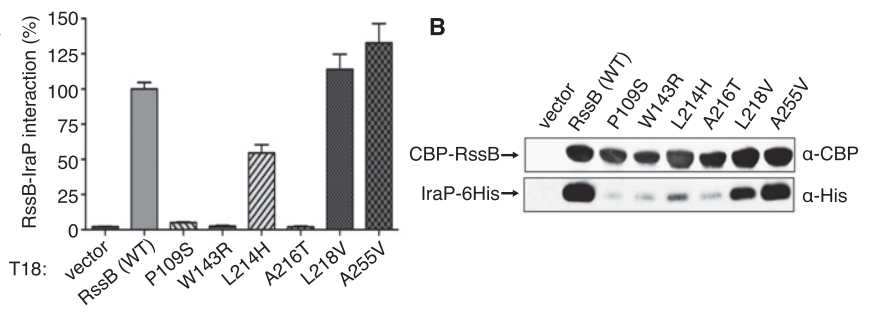

c
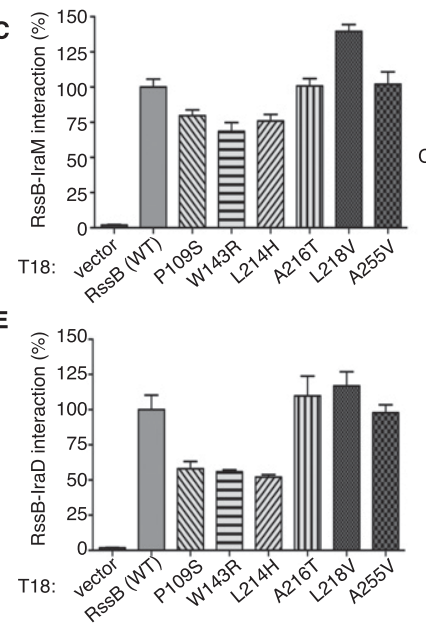

D
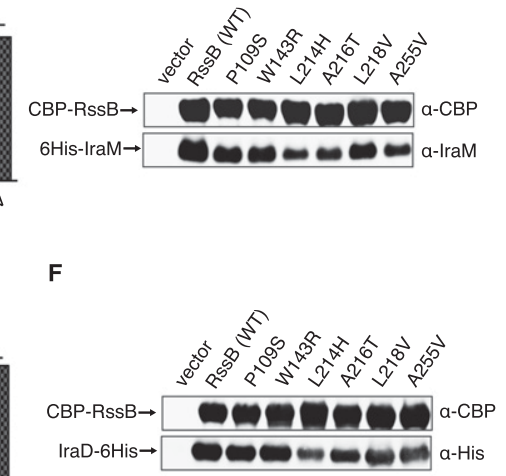

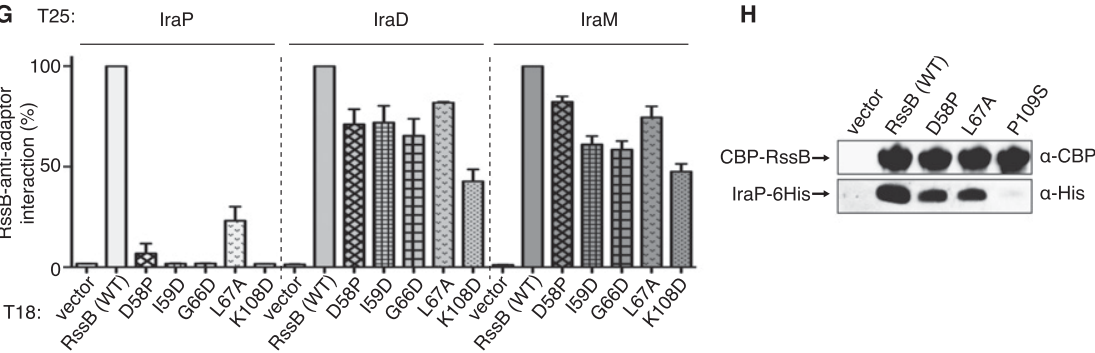

Figure 5. Interaction between the RssB mutants and anti-adaptor proteins. In $A$ (IraP), $C$ (IraM), $E$ (IraD), and $G$, the indicated RssB alleles fused to the T18 domain were assayed for interaction with the anti-adaptor proteins fused to the T25 domain in the BTH101 strain, as described in the Materials and Methods. The value of the interaction between RssB wild-type (WT) and anti-adaptor proteins was set at $100 \%$ for each series. Mean values $(n=3)$ obtained for each interaction in Miller units were (see Supplemental Table S1) as follows: 1370 for T25IraP/T18-RssB, 1222 for T25-IraD/T18-RssB, and 1641 for T25-IraM/T18-RssB. Values $(n=3)$ obtained for each interaction in $G$ in Miller units were as follows: 3593 for T25IraP/T18-RssB, 4269 for T25-IraD/T18-RssB, and 5144 for T25-IraM/T18-RssB. In $B$ (IraP), $D$ (IraM), $F(\operatorname{IraD})$, and $H$, an in vivo pulldown assay was used to measure interaction between the RssB mutants and the antiadaptor proteins as in Figure 1C. Samples were analyzed by Western blot using antiCBP, anti-His, and anti-IraM antisera.
Supplemental Table S5). It is also the only anti-adaptorresistant mutant in the response regulator domain and has the characteristics we expected of a mutation disrupting the IraP interaction surface. In both the twohybrid and pull-down assays, there was no detectable interaction between P109S and IraP (Fig. 5A,B). However, P109S retained the ability to interact with IraM (Fig. $5 \mathrm{C}, \mathrm{D}$ ) or $\operatorname{IraD}$ (Fig. 5E,F), the anti-adaptors to which it is sensitive. The isolation of an IraP-resistant mutant in the response regulator domain is fully consistent with our finding that IraP interacts with the RssB response regulator domain (Fig. 1). Furthermore, because P109S retains both the interaction with IraD and sensitivity to IraD, the interaction surfaces for IraP and IraD in the response regulator domain cannot completely overlap.

As noted above, a mutation in the response regulator domain that blocks phosphorylation was still sensitive to anti-adaptors (Supplemental Fig. S5A), while P109S, close to critical residues for phosphorylation, was resistant to IraP. We constructed several mutants in and around the phosphorylation site (Fig. 2G) and tested them for their ability to interact with the anti-adaptors. Unlike P109S, mutants that block phosphorylation (for instance, D58P,
L67A, and K108D) (Fig. 4A; data not shown) were still sensitive to IraP (Supplemental Fig. S5B). None of the mutants disrupted the interaction with IraD or IraM (Fig. 5G,H). However, while some mutants showed normal interaction with IraP (in gray in Fig. 2F), several-including D58P and L67A, both of which were not detectably phosphorylated in vitro (Fig. 4A)-exhibited decreased interaction with IraP in the bacterial two-hybrid assay (Fig. 5G) but not in a pull-down assay (Fig. 5H). Overall, the results suggest that phosphorylation of RssB improves the ability of IraP to interact but is not essential for interaction (as measured in the pull-down assay) or inhibition (Supplemental Fig. S5). The bacterial twohybrid assay may be somewhat more sensitive than the direct interaction (pull-down) assay to perturbations in the interaction of IraP and RssB.

W143R, in the linker, is resistant to both IraP and IraD but sensitive to IraM (Figs. 2, 3; Supplemental Table S5). Like P109S, it has lost interaction with IraP (Fig. 5A,B) but retains interactions with IraM (Fig. 5C,D). In fact, both P109S and W143R appear to be hypersensitive to IraM in vivo (Fig. 2D) and in vitro (Fig. 3D). W143R may act to change accessibility of the $\mathrm{N}$-terminal domain to IraP and 
IraD. This change is sufficient to block IraP binding but does not block binding of IraD in the in vivo test. The conformation of RssB in W143R appears to be particularly favorable for IraM action.

\section{Anti-adaptor-resistant mutants in the C-terminal domain of RssB do not disrupt anti-adaptor binding}

The four C-terminal RssB mutants are resistant to all three anti-adaptors in vitro (Fig. 3). However, unlike the mutations in the response regulator domain and linker, three mutants-L214H, A218V, and A255V-retained the ability to interact with all of the anti-adaptors (Fig. 5). Therefore, these residues are unlikely to define the interaction surfaces for the anti-adaptors. L218V, which is resistant to IraP, illustrates this point; the mutation is within the C-terminal effector domain, whereas IraP interacts with the N-terminal domain (Fig. 1).

How can these mutations be resistant to anti-adaptors but still interact with them? One possibility is that these mutants favor the interaction with RpoS. Consistent with this model, $\mathrm{L} 218 \mathrm{~V}$ and $\mathrm{A} 255 \mathrm{~V}$ behave as if they are constitutively activated for RpoS degradation; that is, they are able to degrade RpoS even in the absence of AcP (Fig. 3B). This result suggests that these mutations cause a shift in RssB conformation to the activated form, akin to what is brought about by phosphorylation. This active conformation of RssB is able to interact with RpoS (Fig. 4D).

A216T, which otherwise acts similarly to L218V and A255V, has lost the ability to interact with IraP; the basis for this phenotype is not yet clear. One possibility is that this mutational change has two independent effects: first, activation of RssB (like L218V), and second, interference with IraP binding.

\section{Wild-type RssB and anti-adaptor-resistant RssB mutants form oligomers}

Many response regulators oligomerize, and there is frequently a transition in the oligomeric state that correlates with activation of the response regulator by phosphorylation (see Gao and Stock 2009, 2010). We queried the ability of RssB to oligomerize using the bacterial twohybrid assay (Supplemental Fig. S6). All six of our antiadaptor-resistant mutants retained the ability to oligomerize in this assay. However, with this assay, the size and nature of the oligomer cannot be determined. In addition, these interactions were only detectable in clp mutants (Supplemental Fig. S6; see the Supplemental Material for further discussion). RssB mutants with amino acid substitutions at or around the site of phosphorylation were generally defective for oligomerization (Supplemental Fig. S6B), and this correlated with their decreased ability to interact with IraP in the bacterial two-hybrid assay (Fig. 5A). It is likely that, like D58P and L67A (Fig. 4A), all of these oligomerization-defective mutants are also defective for phosphorylation. The correlation between lack of phosphorylation and inability to oligomerize is difficult to interpret, given the limits of the bacterial two-hybrid assay. Therefore, the role of oligomers has not been further explored here or incorporated in our model for RssB function, although it is clear that intermolecular RssB interactions are likely to play a role in regulating activity.

\section{Anti-adaptors respond differently to changes at the RssB phosphorylation site}

The reduced interaction of IraP with RssB mutants near the phosphorylation site (Fig. 5G) could mean that IraP favors an activated form of RssB. This was also suggested by the behavior of P109S, a mutant that loses all interaction with IraP (Fig. 5A,B) and is also defective for interaction with RpoS (Fig. 4B,C). IraM and IraD, on the other hand, interacted well with mutants that disrupt phosphorylation (Fig. 5G). In addition, the P109S mutant was hypersensitive to IraM and IraD (Figs. 2D, 3). One interpretation of these results is that IraM and IraD interact with and stabilize an inactive form of RssB that is unable to bind RpoS.

In order to examine this further, we made use of two mutations in the conserved site of phosphorylation: D58P and D58E. Changes of this aspartate to glutamate have been described as phosphorylation mimics in many response regulators (Klose et al. 1993; Domian et al. 1997; Lan and Igo 1998). In our assays, D58E had $\sim 50 \%$ of the activity of wild-type RssB in the presence of AcP and very little response to AcP (data not shown). Thus, while all of our evidence suggests that equilibria between active and inactive forms exist for all of our mutants, D58P should be more "inactive" than wild-type RssB, while D58E should be more "active." The sensitivity of these mutants to the anti-adaptors was determined and compared with wild-type RssB (Supplemental Fig. S7). While the sensitivity of these three forms of RssB to IraP was essentially identical, D58P was significantly more sensitive to IraD and IraM than wild-type RssB, while D58E was significantly more resistant to IraD and modestly more resistant to IraM. This supports a model in which IraM and IraD prefer the inactive form of RssB.

\section{Discussion}

E. coli and its relatives use RpoS to mount a global response to multiple stresses. Too little or too much RpoS is harmful, and complex and overlapping regulatory schemes control RpoS levels (for review, see Battesti et al. 2011). The findings here increase our understanding of how the proteolytic branch of these regulatory pathways works. The unusual response regulator RssB allows the system to receive inputs from multiple anti-adaptors, regulating RpoS degradation. As the cells encounter a stress or enter stationary phase, induction of the antiadaptors provides a mechanism for rapidly stabilizing RpoS; other signaling pathways, via sRNAs, lead to increased translation of RpoS. The low level of RssB ensures the ability of the anti-adaptors to fully titrate RssB. Because the anti-adaptors are not themselves subject to degradation, they will continue to act until no 
longer needed. When the stress passes, the system resets itself in a process that has not yet been fully studied.

Our studies of RssB alleles resistant to an anti-adaptor have provided insight into both how the anti-adaptors act and how RssB functions. A summary of the assays performed with each of the mutants is provided in Table 1 (see Supplemental Table S5 for a more detailed summary). Based on the results of these and previous studies, we propose the model for RssB function and its inhibition by anti-adaptors shown in Figure 6 and discussed below. While each of the six mutants studied here behaves in a distinct manner, we propose that they fall into two major classes and show how they likely affect RssB in Figure 6. In our model, IraD and IraM interact with inactive RssB, blocking its ability to interact with RpoS and deliver RpoS to the protease. IraP, on the other hand, may mimic RpoS in its interaction with RssB, preferring the active form of RssB and directly competing with RpoS for binding to the $\mathrm{N}$-terminal domain. Phosphorylation and RpoS binding both help to stabilize the activated form of RssB. Class I mutants, in the response regulator domain, specifically interfere with the ability of IraP to interact with RssB. Class II mutants lie in the C-terminal PP2C-like domain of RssB and activate the protein, bypassing the need for AcP.

Mutants in the response regulator domain of RssB define a region important for IraP interaction: class I RssB mutants

A variety of evidence supports the interaction of IraP primarily with the response regulator domain. Bacterial two-hybrid interactions and pull-down experiments demonstrate interactions with the response regulator domain and not with the C-terminal domain (Fig. 1). P109S, within the response regulator domain, is specifically resistant to IraP, while it becomes hypersensitive to IraM and IraD (Figs. 2, 3; Supplemental Table S5) and loses interactions with IraP (Fig. 5). Mutants such as P109S that specifically block IraP binding are defined as class I (Fig. 6).
In the crystal structure of the $\mathrm{N}$-terminal domain of RssB (Fig. 2G), P109 lies on the surface, not far from the position of D58, the site of phosphorylation (Bouché et al. 1998). K108, P109, and V110 form a conserved motif found in all response regulators; K108 is necessary for activation, while P109 affects the orientation of the $\beta 5 / \alpha 5$ face of the response regulator (Volz 1995; Bourret 2010), a region implicated in protein-protein interactions for both dimerization and ligand binding (Gao and Stock 2010). We suggest that IraP interacts with this face of RssB, interfering with its interactions with its ligand, RpoS (Fig. 6); the mutant disrupts this interaction. P109S, although selected to retain its ability to function in RpoS degradation, shows decreased interaction with RpoS (Fig. 4C) and loss of RpoS-dependent formation of an NEMresistant complex (Fig. 4B). Degradation of RpoS by P109S is only slightly defective, compared with wild-type RssB (Fig. 3A), suggesting that the defect in interaction of P109S and RpoS can be suppressed by formation of a larger complex with ClpXP. P109 may not contact IraP directly; if the binding sites for IraP and RpoS on RssB overlap, a mutant preventing IraP binding might have failed our screen, since it would be defective for RpoS binding and degradation.

\section{The role of RssB phosphorylation}

Unlike many other response regulators, RssB retains significant activity in the absence of phosphorylation, suggesting that while phosphorylation may increase the amount of the protein in an active conformation, this switch from inactive to active is not absolutely dependent on phosphorylation (see active forms of RssB in Fig. 6). Our data clearly demonstrate that the status of phosphorylation, per se, is not necessary for any of the anti-adaptors to act. Anti-adaptors can inhibit the wildtype RssB in the absence of phosphorylation by AcP (Supplemental Fig. S4) and can also inhibit the RssB mutant at the site of phosphorylation (Supplemental Fig. S5).

Table 1. Summary of RssB mutant phenotypes

\begin{tabular}{|c|c|c|c|c|c|c|c|}
\hline RssB & Wild type & P109S & W143R & L214H & $\mathrm{A} 216 \mathrm{~T}$ & L218V & A255V \\
\hline Activity & +++ & ++ & +++ & +++ & ++++ & ++++ & ++++ \\
\hline AcP-dependent? & Yes & Yes & Yes & Yes & No & No & No \\
\hline Phosphorylated? & Yes & Low & Yes & Yes & Yes & Yes & Yes \\
\hline NEM-resistant? & Yes & No & Yes & Yes & Yes & Yes & Yes \\
\hline \multicolumn{8}{|l|}{ IraP } \\
\hline Activity & S & $\mathrm{R}$ & $\mathrm{R}$ & $\mathrm{R}$ & $\mathrm{R}$ & $\mathrm{R}$ & $\mathrm{R} / \mathrm{S}^{\mathrm{a}}$ \\
\hline Binding & Yes & No & No & Yes & No & Yes & Yes \\
\hline \multicolumn{8}{|l|}{ IraD } \\
\hline Activity & $S$ & $S$ & $\mathrm{R}$ & $\mathrm{R}$ & $\mathrm{R}$ & $\mathrm{R}$ & $\mathrm{R} / \mathrm{S}^{\mathrm{a}}$ \\
\hline Binding & Yes & Yes & Yes & Yes & Yes & Yes & Yes \\
\hline \multicolumn{8}{|l|}{ IraM } \\
\hline Activity & $S$ & $S$ & $S$ & $S$ & $\mathrm{R}$ & $\mathrm{R}$ & $\mathrm{R}$ \\
\hline Binding & Yes & Yes & Yes & Yes & Yes & Yes & Yes \\
\hline
\end{tabular}

(R) Resistant; (S) sensitive. Results are compiled from Figures 1-5. A more detailed summary, with quantitations, is in Supplemental Table S5.

${ }^{\mathrm{a}} \mathrm{A} 255 \mathrm{~V}$ is resistant to $\mathrm{IraP}$ and $\mathrm{IraD}$ in vitro but is not significantly resistant in vivo. 


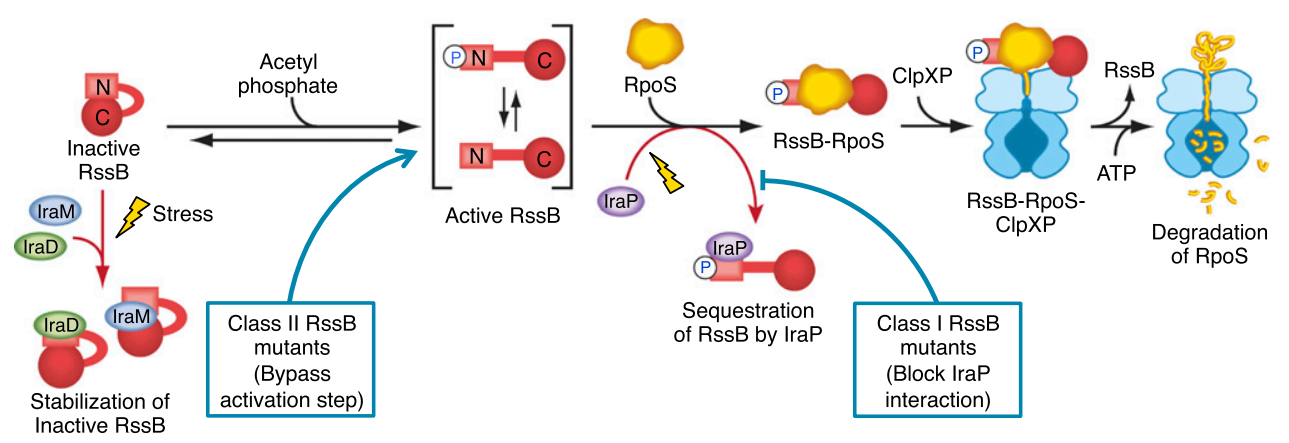

Figure 6. Model for RssB function based on anti-adaptor-resistant mutants. In the figure, RssB is represented in multiple conformations. (1) "Inactive" form: This form is shown as an inactive monomer. We suggest that the $\mathrm{N}$ terminus and $\mathrm{C}$ terminus of RssB interact in this conformation. (2) "Active" forms: These forms are stabilized by phosphorylation and/or RpoS binding. In this model, we suggest the existence of an active and reversible state that may be promoted by RpoS binding and/or RssB phosphorylation. This is shown here as a monomer but could be a dimer as well. Mutations that cannot be phosphorylated have a lower level of activated RssB but are still able to present RpoS to the protease. The stable and active form of RssB, phosphorylated and bound to RpoS, is formed under optimal conditions. Based on the interactions defined here, both IraD and IraM can interact with the inactive form of RssB, stabilizing an inactive conformation that is unable to interact with RpoS (shown at the left). They are also able to interact with the active form (activated class II RssB mutants), but that interaction does not result in inhibition of RssB activity. These two anti-adaptors act by different mechanisms; IraD interacts with the $\mathrm{N}$-terminal domain, and IraM interacts primarily with the C-terminal domain. IraP interacts with both active forms of RssB but favors the phosphorylated form. Mutants from class I block the IraP interaction. Mutants from class II bypass or partially bypass the need for phosphorylation, increasing the population of "active" RssB and improving the ability of RssB to interact with RpoS.

Although phosphorylation is associated with increased activity of RssB and is not required for IraP to act, our data suggest that IraP interacts preferentially with the "activated" form of RssB (Fig. 6). This is based primarily on the decreased interaction of mutations that block phosphorylation-D58P and L67A-with IraP (Fig. 5G). It is not clear how strong this preference is, since IraP inhibits wild-type RssB, D58P, and D58E similarly (Supplemental Fig. S7). Our model is consistent with phosphorylation favoring changes in the ligand-binding face to promote ligand binding (RpoS or IraP).

While not directly addressed here, we suggest that RpoS is likely to stimulate phosphorylation of the response regulator RssB. Ligand stimulation of phosphorylation has been seen for other response regulators (Ames et al. 1999; Schuster et al. 2001; Boulanger et al. 2013). It has previously been shown (and confirmed here) that phosphorylation of RssB stimulates RpoS degradation by ClpXP (Zhou et al. 2001). This positive regulatory loop should lead to rapid removal of RpoS if any source of phosphorylation is available. This may be particularly important in reducing RpoS levels as the bacteria recover from stress or stationary phase and resume rapid growth.

\section{Mutants in the C-terminal effector domain of RssB activate RssB without phosphorylation: class II RssB mutants}

In many response regulators, the response regulator domain and effector domain interact in the absence of phosphorylation, inhibiting activity of the C-terminal effector domain; phosphorylation releases this inhibition (Gao and Stock 2010). We suggest that this is the case for inactive RssB (Fig. 6), although we do not yet have evidence for the direct interaction of the $\mathrm{N}$-terminal and
C-terminal domains. Activation is predicted to free the domains to bind their new partners: RpoS and ClpX. IraP, with its preference for the phosphorylated form of RssB, may block RpoS degradation by competing with RpoS for binding to the freed RssB response regulator domain (Fig. 6). IraM, with contacts in both the $\mathrm{N}$ terminus and C terminus (Fig. 1), may help keep the N-terminal and C-terminal domains in the inactive conformation, possibly even bridging $\mathrm{N}$-terminal and C-terminal domains (Fig. 6). IraD may have a similar ability to stabilize the inactive conformation by interactions with the $\mathrm{N}$ terminus (Fig. 6). Both IraD and IraM preferentially inhibit RssB in the "inactive" D58P form compared with the wild type or the active D58E form (Supplemental Fig. S7), consistent with these anti-adaptors preferring the inactive form of RssB. IraD may also act in a qualitatively different manner from IraP and IraM, given the significantly lower levels of IraD needed to inhibit RpoS degradation in vitro (Supplemental Figs. S4, S7). In every case, the anti-adaptors interfere with the ability of RpoS to interact with RssB (Fig. 1D). How, then, do our C-terminal mutants escape each of these different anti-adaptors? We focus here on the two most C-terminal mutants: L218V and A255V. Both were selected as IraM-resistant (Supplemental Table S3) and are also resistant to IraP and IraD in vitro; $\mathrm{L} 218 \mathrm{~V}$ is resistant to IraP and IraD in vivo as well (Table 1; Figs. 2, 3; Supplemental Fig. S3). These mutant RssB proteins act as if they are constitutively activated, causing a global change that mimics the change brought about by phosphorylation. Most strikingly, they are independent of AcP for degradation of RpoS. In addition, they are partially resistant to NEM in the absence of RpoS, suggesting that activation is not simply tighter binding to RpoS (Fig. 4B). Activation allows them to be resistant to multiple anti-adaptors while retaining interactions with those anti-adaptors, possibly 
by improving binding to RpoS. These mutants thus also provide a window into why RpoS degradation is as complex as it is. If RssB were poised in an "activated" form in vivo, it might behave more like our class II mutants, resistant to anti-adaptors.

The remaining two mutants, $\mathrm{L} 214 \mathrm{H}$ and A216T, have some but not all of the properties of L218V and may represent weaker alleles that affect similar steps, possibly requiring the interaction of RpoS to drive them into the fully active conformation. In addition to the mutant proteins studied in depth here, additional anti-adaptorresistant mutants were identified in this C-terminal region, close to positions 214, 216, and 218 (Supplemental Table S4). A221T had properties similar to $\mathrm{L} 214 \mathrm{H}$. L222W, isolated as IraM-resistant, had properties much like those of L218V (IraP and IraM resistance, with partial resistance to IraD) (Supplemental Fig. S3A,D,E). A215G (Supplemental Table S4) was isolated as IraP-resistant in the context of Q254K and a third mutation (Supplemental Fig. S2A; Supplemental Table S2); both A215G and Q254K were IraD-resistant on plates (Supplemental Fig. S2D), and the double A215G Q254K mutation was also partially resistant to IraM (Supplemental Table S4). The properties of these single and double mutants, as assessed in vivo by the plate assay, support the similarity of these C-terminal mutants to the class II mutants.

\section{A conserved signaling helix in PP2C domains and the RssB effector domain}

The RssB C terminus is predicted to encode an inactive PP2C phosphatase; a well-conserved patch of amino acids from 214 to 222 encodes a helix in these proteins (Galperin 2006). Activating mutations were also found in this helix in a distantly related bacterial PP2C signaling protein, RsbP (Brody et al. 2009). RsbP is a phosphatase involved in the general stress response in Bacillus subtilis. Activation of the phosphatase activity occurs upon energy stress and is dependent on both its own N-terminal domains and a hydrolase, encoded by rsbQ. Brody et al. (2009) selected $r s b P$ suppressors of a deletion of the gene for the activating hydrolase. The majority of these suppressors fell into two helices: $\alpha 0$ and $\alpha 1$ (Brody et al. 2009). Our cluster of mutations falls in the middle of $\alpha 1$ and, like the mutations in RsbP, appears to bypass the need for activation to varying degrees. The $\alpha 0$ and $\alpha 1$ mutations were suggested to define sites of allostery, receiving signals to control phosphatase activity (Brody et al. 2009). In our case, the "output" of RssB is reversed-not phosphatase activity, but loss of inhibition of the N-terminal domain and ability to interact with ClpX to deliver RpoS to the protease. Supplemental Figure S8 shows the alignments of RsbP and RssB and the position of constitutive mutants in both (Supplemental Fig. S8A) as well as parallels between these signaling cascades (Supplemental Fig. S8B).

\section{Summary}

The large set of anti-adaptor-resistant RssB mutants that we isolated has allowed us to explore the mechanism of action of the RssB adaptor protein and its inhibition by anti-adaptors. IraP interacts with the conserved response regulator domain, probably directly competing with RpoS for access to RssB (Fig. 6). The interaction of IraP with this well-conserved response regulator domain suggests that other response regulators might be inhibited in a similar fashion by related proteins. However, these interactions would have to show specificity for the right response regulator. A search for proteins related to IraP has not uncovered such a family, but a better understanding of the interaction surface on IraP may allow a more informed search.

IraD also interacts with the response regulator domain but in a mode that is distinct from the interaction of IraP. IraM has a third and distinct mode of interaction, primarily with the C-terminal domain; contacts have not yet been defined by any of our mutations.

For most response regulators, the major input for regulatory signaling is via phosphorylation of the conserved aspartate (for review, see Gao and Stock 2009). This is clearly not true for RssB. Phosphorylation, while stimulatory, is not essential for RssB to function or for the response to environmental signals (Fig. 3A; Peterson et al. 2004). Therefore, other inputs are needed to regulate outcome, and the small protein anti-adaptors provide at least some of these additional inputs, blocking RpoS degradation by interactions with both the active (IraP) and inactive (IraD and IraM) forms of RssB (Fig. 6). There is every reason to believe that similar small proteins will interact with and modulate the activity of other complex response regulators.

\section{Materials and methods}

\section{Media and growth conditions}

Cells were grown in LB (Lennox broth). Plasmids were maintained with $100 \mu \mathrm{g} / \mathrm{mL}$ ampicillin, $50 \mu \mathrm{g} / \mathrm{mL}$ kanamycin, or $30 \mu \mathrm{g} / \mathrm{mL}$ chloramphenicol. Liquid cultures were grown under aerobic conditions at $37^{\circ} \mathrm{C}$ for RpoS stability and pull-down experiments and at $32^{\circ} \mathrm{C}$ for the $\beta$-galactosidase assays unless stated otherwise. Lactose MacConkey agar plates were used in analyses of strains carrying rpoS-lacZ fusions.

\section{Strains and plasmids}

Strains are listed in Supplemental Table S6, plasmids are listed in Supplemental Table S7, and oligonucleotides are listed in Supplemental Table S8. All strains are derivatives of E. coli K12. Bacterial two-hybrid experiments were carried out in BTH101 (Karimova et al. 1998) or derivatives constructed by P1 transduction. The native promoters of iraP, iraM, and iraD were replaced with synthetic constitutive promoters (Cp18 for iraP, Cp13 or Cp18 for iraM, and Cp26 for iraD) (Jensen and Hammer 1998) tagged with a zeocin resistance marker for selection using the bacteriophage $\lambda$ red recombination system as described in the Supplemental Material.

To construct pET-IraM, an E. coli codon-optimized iraM gene fragment was synthesized (IDT) (Supplemental Table S8) and inserted into pET151/D-TOPO (Invitrogen) using directional topoisomerase cloning as recommended by the manufacturer.

\section{Library screening}

Random mutagenesis of RssB was performed at a low mutation rate (two to nine mutations per kilobase) using the GeneMorph II 
EZClone domain mutagenesis kit (Stratagene) according to the manufacturer's specifications. Details are described in the Supplemental Material. The results of the screen are summarized in Supplemental Tables S2 and S3; mutant plasmids were found at a rate of $0.2 \%$. Each mutant was reconstructed as the multiple and each single mutation using the QuickChange II site-directed mutagenesis kit (Stratagene). Behavior of the original and reconstructed mutants are summarized in Supplemental Table S4 and discussed further in the Supplemental Material.

\section{Assay for RpoS degradation in vivo}

Cells were grown overnight in LB medium, diluted into fresh LB medium at an $\mathrm{OD}_{600} \approx 0.01$, and grown to mid-logarithmic phase $\left(\mathrm{OD}_{600} \approx 0.3\right)$ at $37^{\circ} \mathrm{C}$. Chloramphenicol was added $(200 \mu \mathrm{g} / \mathrm{mL})$. Samples $(1 \mathrm{~mL})$ were harvested at the indicated time points and precipitated with a final concentration of $5 \%$ ice-cold trichloroacetic acid (TCA). Precipitated pellets were washed with $500 \mu \mathrm{L}$ of $80 \%$ cold acetone, dried, and resuspended in a volume of SDS sample buffer normalized to the $\mathrm{OD}_{600}$.

\section{In vivo pull-down experiments}

pACYC184-CBP plasmids encoding RssB or RssB domains were cotransformed with pQE80L plasmids encoding the anti-adaptors, grown with antibiotic selection, and induced for $1 \mathrm{~h}$. Cells were collected, lysed, incubated with calmodulin beads, and washed, and samples associated with the beads were analyzed by Western blotting following protein electrophoresis (for detailed methods, see the Supplemental Material).

\section{Protein electrophoresis and Western blotting}

Samples were analyzed using Nu-PAGE $12 \%$ Bis-Tris gels (Invitrogen), transferred to nitrocellulose membrane, and probed with a 1:5000 dilution of anti-RpoS anti-serum, a 1:1000 dilution of anti-CBP anti-serum (Millipore), a 1:1000 dilution of anti-His antiserum (Santa Cruz Biotechnology), or a 1:2000 dilution of antiIraM antiserum. The blots were developed with the Lumi-Phos WB chemiluminescent substrate (Thermo Scientific) using the Luminescent Image Analyzer LAS-4000 Mini (Fujifilm). Quantification was performed by using Multi-Gauge software (Fujifilm). Values presented are the mean of at least three independent assays.

\section{Bacterial two-hybrid assays}

We used the adenylate cyclase-based bacterial two-hybrid system (Karimova et al. 1998). Plasmids containing the pair of proteins to be tested fused to the T18 and T25 domains of the adenylate cyclase from $B$. pertussis were cotransformed in BTH101 or derivatives, plated on plates containing $100 \mu \mathrm{g} / \mathrm{mL}$ ampicillin and $50 \mu \mathrm{g} / \mathrm{mL}$ kanamycin, and incubated for $48 \mathrm{~h}$ at $30^{\circ} \mathrm{C}$. Cells were grown overnight in $3 \mathrm{~mL}$ of $\mathrm{LB}$ medium supplemented with $100 \mu \mathrm{g} / \mathrm{mL}$ ampicillin, $50 \mu \mathrm{g} / \mathrm{mL}$ kanamycin, and $0.5 \mathrm{mM}$ IPTG at $32^{\circ} \mathrm{C}$. $\beta$-Galactosidase activity was determined using the standard assay described by Miller (1992). Values presented are the mean of at least three independent assays.

\section{Protein purification}

RssB wild-type or mutant protein was purified after expression from a T7 promoter as described in the Supplemental Materials. $\mathrm{IraP}_{-\mathrm{His}}{ }_{6}$ and $\mathrm{IraD}^{-\mathrm{His}_{6}}$ were overexpressed and purified as described (Bougdour et al. 2006, 2008) with slight modifications (see the Supplemental Material). His 6 -IraM was overexpressed and purified from pET-IraM as described in the Supplemental Material.

\section{RpoS in vitro degradation}

In vitro RpoS degradation was performed essentially as described (Zhou et al. 2001). Reaction mixtures were assembled in $30 \mu \mathrm{L}$ of buffer B (20 mM Tris-HCl at pH 7.5, $10 \mathrm{mM} \mathrm{MgCl}_{2}, 100 \mathrm{mM} \mathrm{KCl}$, $5 \mathrm{mM}$ DTT, $0.1 \mathrm{mM}$ EDTA, $5 \%$ glycerol [v/v]) containing $5 \mathrm{mM}$ ATP, $25 \mathrm{mM}$ AcP, $0.1 \mu \mathrm{M}$ ClpX, $0.2 \mu \mathrm{M}$ ClpP, $1.8 \mu \mathrm{M}[3 \mathrm{H}] \mathrm{RpoS}$, and $0.4 \mu \mathrm{M}$ RssB unless otherwise indicated. IraP $(1 \mu \mathrm{M})$, IraM $(1 \mu \mathrm{M})$, or $\operatorname{IraD}(0.18 \mu \mathrm{M})$ was added as indicated. The mixtures were incubated for $20 \mathrm{~min}$ at $23^{\circ} \mathrm{C}$. TCA was added to $15 \%(\mathrm{w} / \mathrm{v})$, and $\left[{ }^{3} \mathrm{H}\right]$ RpoS degradation was quantitated by measuring acidsoluble radioactivity.

\section{NEM sensitivity}

Reaction mixtures containing 1.2 $\mu \mathrm{M}$ RssB wild type or mutant were assembled in $10 \mu \mathrm{L}$ of buffer B in the presence and absence of $25 \mathrm{mM} \mathrm{AcP}$ and/or 5.4 $\mu \mathrm{M} \mathrm{RpoS}$ as indicated and incubated for $5 \mathrm{~min}$ at $23^{\circ} \mathrm{C}$. NEM was added to $10 \mathrm{mM}$, incubated $10 \mathrm{~min}$ at $23^{\circ} \mathrm{C}$, and then neutralized for $10 \mathrm{~min}$ at $23^{\circ} \mathrm{C}$ by the addition of $20 \mathrm{mM}$ DTT. RssB NEM sensitivity was assessed by measuring RpoS degradation by ClpXP as described above.

\section{Mobility shift detection of phosphorylated RssB on Phos-tag SDS-PAGE gel}

Purified wild-type or mutant RssB was incubated in the presence or absence of $20 \mathrm{mM} \mathrm{AcP}$ for $30 \mathrm{~min}$ at room temperature, SDS sample buffer was added, and samples were separated and visualized on polyacrylamide gels containing Phos-tag as previously described (described in the Supplemental Material; Barbieri and Stock 2008; Kinoshita and Kinoshita-Kikuta 2011).

\section{Acknowledgments}

We thank members of the laboratory, M. Galperin, M. Maurizi, and D. Dougan, for their comments on the manuscript. We thank A. Boulanger for discussions on using the Phos-tag method. This research was supported by the Intramural Research Program of the National Institutes of Health (NIH), National Cancer Institute, Center for Cancer Research. J.M.M. was supported by the $\mathrm{NIH}$ undergraduate scholarship program.

\section{References}

Ames SK, Frankema N, Kenney LJ. 1999. C-terminal DNA binding stimulates $\mathrm{N}$-terminal phosphorylation of the outer membrane protein regulator OmpR from Escherichia coli. Proc Natl Acad Sci 96: 11792-11797.

Barbieri CM, Stock AM. 2008. Universally applicable methods for monitoring response regulator aspartate phosphorylation both in vitro and in vivo using Phos-tag-based reagents. Anal Biochem 376: 73-82.

Battesti A, Bouveret E. 2012. The bacterial two-hybrid system based on adenylate cyclase reconstitution in Escherichia coli. Methods 58: 325-334.

Battesti A, Majdalani N, Gottesman S. 2011. The RpoS-mediated general stress response in Escherichia coli. Annu Rev Microbiol 65: 189-213.

Becker G, Klauck E, Hengge-Aronis R. 2000. The response regulator RssB, a recognition factor for $\sigma^{\mathrm{S}}$ proteolysis in 
Escherichia coli, can act like an anti- $\sigma^{\mathrm{S}}$ factor. Mol Microbiol 35: 657-666.

Bouché S, Klauck E, Fischer D, Lucassen M, Jung K, HenggeAronis R. 1998. Regulation of RssB-dependent proteolysis in Escherichia coli: A role for acetyl phosphate in a response regulator-controlled process. Mol Microbiol 27: 787-795.

Bougdour A, Wickner S, Gottesman S. 2006. Modulating RssB activity: IraP, a novel regulator of $\sigma^{\mathrm{S}}$ stability in Escherichia coli. Genes Dev 20: 884-897.

Bougdour A, Cunning C, Baptiste PJ, Elliott T, Gottesman S. 2008. Multiple pathways for regulation of $\sigma^{\mathrm{S}}$ (RpoS) stability in Escherichia coli via the action of multiple anti-adaptors. Mol Microbiol 68: 298-313.

Boulanger A, Chen Q, Hinton DM, Stibitz S. 2013. In vivo phosphorylation dynamics of the Bordetella pertussis virulence-controlling response regulator BvgA. Mol Microbiol 88: 156-172.

Bourret RB. 2010. Receiver domain structure and function in response regulator proteins. Curr Opin Microbiol 13: 142149.

Brody MS, Stewart V, Price CW. 2009. Bypass suppression analysis maps the signalling pathway within a multidomain protein: The RsbP energy stress phosphatase 2C from Bacillus subtilis. Mol Microbiol 72: 1221-1234.

Domian IJ, Quon KC, Shapiro L. 1997. Cell type-specific phosphorylation and proteolysis of a transcriptional regulator controls the G1-to-S transition in a bacterial cell cycle. Cell 90: 415-424.

Galperin MY. 2006. Structural classification of bacterial response regulators: Diversity of output domains and domain combinations. J Bacteriol 188: 4169-4182.

Galperin MY. 2010. Diversity of structure and function of response regulator output domains. Curr Opin Microbiol 13: $150-159$.

Gao R, Stock AM. 2009. Biological insights from structures of two-component proteins. Annu Rev Microbiol 63: 133-154.

Gao R, Stock AM. 2010. Molecular strategies for phosphorylation-mediated regulation of response regulator activity. Curr Opin Microbiol 13: 160-167.

Gully D, Bouveret E. 2006. A protein network for phospholipid synthesis uncovered by a variant of the tandem affinity purification method in Escherichia coli. Proteomics 6: 282293.

Hengge R. 2009. Proteolysis of $\sigma^{\mathrm{S}}$ (RpoS) and the general stress response in Escherichia coli. Res Microbiol 160: 667-676.

Jensen PR, Hammer K. 1998. The sequence of spacers between the consensus sequences modulates the strength of prokaryotic promoters. Appl Environ Microbiol 64: 82-87.

Karimova G, Pidoux J, Ullmann A, Ladant D. 1998. A bacterial two-hybrid system based on a reconstituted signal transduction pathway. Proc Nat1 Acad Sci 95: 5752-5756.

Kinoshita E, Kinoshita-Kikuta E. 2011. Improved phos-tag SDSPAGE under neutral $\mathrm{pH}$ conditions for advanced protein phosphorylation profiling. Proteomics 11: 319-323.

Klauck E, Lingnau M, Hengge-Aronis R. 2001. Role of the response regulator RssB in $\sigma^{\mathrm{s}}$ recognition and initiation of $\sigma^{\mathrm{S}}$ proteolysis in Escherichia coli. Mol Microbiol 40: 13811390.

Klose KE, Weiss DS, Kustu S. 1993. Glutamate at the site of phosphorylation of nitrogen-regulatory protein $\mathrm{NtrC}$ mimics aspartl-phosphate and activates the protein. J Mol Biol 232: 67-78.

Lan C-Y, Igo MM. 1998. Differential expression of the OmpF and OmpC porin proteins in Escherichia coli K-12 depends upon the level of active OmpR. J Bacteriol 180: 171-174.
Merrikh H, Ferrazzoli AE, Bougdour A, Olivier-Mason A, Lovett ST. 2009a. A DNA damage response in Escherichia coli involving the alternative $\sigma$ factor RpoS. Proc Natl Acad Sci 106: 611-616.

Merrikh H, Ferrazzoli AE, Lovett ST. 2009b. Growth phase and (p)ppGpp control of IraD, a regulator of rpoS stability, in Escherichia coli. J Bacteriol 191: 7436-7446.

Miller JH. 1992. A short course in bacterial genetics. Cold Spring Harbor Laboratory Press, Cold Spring Harbor, NY.

Peterson CN, Ruiz N, Silhavy TJ. 2004. RpoS proteolysis is regulated by a mechanism that does not require the SprE (RssB) response regulator phosphorylation site. I Bacteriol 186: $7403-7410$.

Schuster M, Silversmity RE, Bourret RB. 2001. Conformational coupling in the chemotaxis response regulator CheY. Proc Natl Acad Sci 98: 6003-6008.

Volz K. 1995. Structural and functional conservation in response regulators. In Two-component signal transduction (ed. Hoch JA and Silhavy TJ), pp. 53-64. ASM Press, Washington, D.C. Zhou Y-N, Gottesman S. 1998. Regulation of proteolysis of the stationary-phase $\sigma$ factor RpoS. J Bacteriol 180: 1154-1158.

Zhou Y, Gottesman S, Hoskins JR, Maurizi MR, Wickner S. 2001. The RssB response regulator directly targets $\sigma^{\mathrm{S}}$ for degradation by ClpXP. Genes Dev 15: 627-637. 


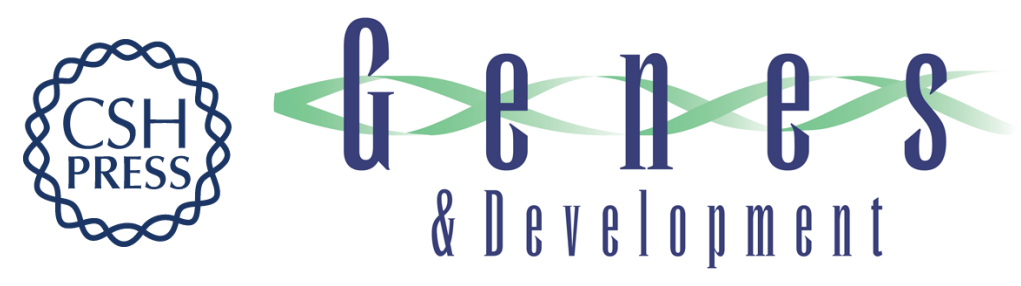

\section{Anti-adaptors provide multiple modes for regulation of the RssB adaptor protein}

Aurelia Battesti, Joel R. Hoskins, Song Tong, et al.

Genes Dev. 2013, 27:

Access the most recent version at doi:10.1101/gad.229617.113

\section{Supplemental http://genesdev.cshlp.org/content/suppl/2013/12/18/27.24.2722.DC1 Material}

References This article cites 30 articles, 12 of which can be accessed free at: http://genesdev.cshlp.org/content/27/24/2722.full.html\#ref-list-1

Creative This article is distributed exclusively by Cold Spring Harbor Laboratory Press for the first Commons six months after the full-issue publication date (see

License http://genesdev.cshlp.org/site/misc/terms.xhtml). After six months, it is available under a Creative Commons License (Attribution-NonCommercial 3.0 Unported), as described at http://creativecommons.org/licenses/by-nc/3.0/.

Email Alerting Receive free email alerts when new articles cite this article - sign up in the box at the top Service right corner of the article or click here.

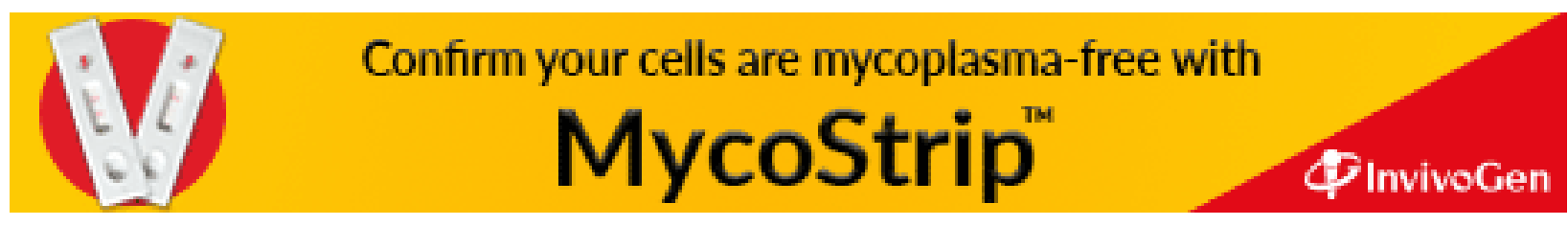

